TRANSACTIONS OF THE

AMERICAN MATHEMATICAL SOCIETY

Volume 358, Number 11, November 2006, Pages 4793-4807

S 0002-9947(06)04059-1

Article electronically published on June 20, 2006

\title{
ON THE CHARACTERIZATION OF THE KERNEL OF THE GEODESIC X-RAY TRANSFORM
}

\author{
EDUARDO CHAPPA
}

\begin{abstract}
Let $\bar{\Omega}$ be a compact manifold with boundary. We consider covariant symmetric tensor fields of order two that belong to a Sobolev space $H^{k}(\bar{\Omega}), k \geq 1$. We prove, under the assumption that the metric is simple, that solenoidal tensor fields that belong to the kernel of the geodesic X-ray transform are smooth up to the boundary. As a corollary we obtain that they form a finite-dimensional set in $H^{k}$.
\end{abstract}

\section{INTRODUCTION}

In this paper we consider the problem of the characterization of the kernel of the geodesic X-ray transform of 2-tensor fields on a compact and simple (see the definition below) Riemannian manifold $\bar{\Omega}$ with metric $g$.

The problem of the characterization of the kernel of the X-ray transform for 2 -forms arises naturally as the linearization of the problem of determining a metric on a compact manifold from its hodograph. We refer the reader to the book by V.A. Sharafutdinov [12] for an explanation of this non-linear problem.

In order to simplify matters we assume that $\bar{\Omega} \subset \mathbb{R}^{n}$ is the closure of an open subset $\Omega$ of $\mathbb{R}^{n}$ and that we have extended $g$ to be a simple Riemannian metric in a neighborhood of $\bar{\Omega}$. We remind the reader that a metric is simple if any two points $p, q \in \bar{\Omega}$ can be joined by a unique geodesic $\gamma_{p q}$ that lies entirely in $\bar{\Omega} \backslash \partial \Omega$ (with the possible exception of only $p$ and $q$ ) and such that $\gamma_{p q}$ depends smoothly on $p$ and $q$. We will use the lowercase letter $e$ to denote the usual Euclidean metric in $\mathbb{R}^{n}$.

For a covariant symmetric 2-tensor field $\omega=\sum_{i, j=1}^{n} \omega_{i j}(x) d x^{i} d x^{j}$, we say that $\omega \in H^{k}(\Omega)$ if $\omega_{i j}$ belongs to the Sobolev space $H^{k}(\Omega)$, for all $i, j$. We denote by $\|\cdot\|_{k ; \Omega}$ the corresponding norm. We write $L^{2}(\Omega)=H^{0}(\Omega)$. When this does not lead to misunderstandings, we omit the domain $\Omega$ in the notation, and simply write $H^{k}$ and $\|\cdot\|_{k}$ for $H^{k}(\Omega)$ and $\|\cdot\|_{k ; \Omega}$, respectively.

Given a covariant 2-tensor $\omega=\sum_{i, j=1}^{n} \omega_{i j}(x) d x^{i} d x^{j} \in H^{k}(\Omega)$, we extend $\omega$ so that it vanishes outside $\Omega$ and define its geodesic X-ray transform by

$$
P_{\gamma} \omega=\int_{-\infty}^{\infty} \sum_{i, j=1}^{n} \omega_{i j}(\gamma(s)) \dot{\gamma}^{i}(s) \dot{\gamma}^{j}(s) d s
$$

Received by the editors December 20, 2002 and, in revised form, August 3, 2004.

2000 Mathematics Subject Classification. Primary 58Jxx; Secondary 44A12, 53Cxx.

This work was partially supported by NSF grant \#DMS-00-70488 and NSF grant \#DMS9705792.

(C)2006 American Mathematical Society Reverts to public domain 28 years from publication 
where $\gamma(s)=\left(\gamma^{1}, \ldots, \gamma^{n}\right)$ is a unit speed geodesic of $\Omega$, with respect to the LeviCivita connection. We denote by $\dot{\gamma}^{i}$ the derivative of $\gamma^{i}$ with respect to the arc length parameter. Note that the above integral is invariantly defined, so the X-ray transform of a covariant two tensor field is well defined.

As stated before, we consider in this paper the question of the characterization of the kernel $Z^{k}$ of $P$ in $H^{k}(k \geq 1)$. In order to motivate our answer, note that if $v=\sum_{i=1}^{n} v_{i} d x^{i} \in H^{k+1}(\Omega)$ is a covariant 1-tensor field, then

$$
\omega=d_{g}^{s} v=\sum_{i, j=1}^{n}\left[\frac{1}{2}\left(\frac{\partial v_{i}}{\partial x_{j}}+\frac{\partial v_{j}}{\partial x_{i}}\right)-\sum_{k=1}^{n} v_{k} \Gamma_{i j}^{k}\right] d x^{i} d x^{j}
$$

belongs to $Z^{k}$ if $\left.v\right|_{\partial \Omega}=0$. Here, and throughout this paper, $\Gamma_{i j}^{k}$ denote the Christoffel symbols associated to the Levi-Civita connection induced by the metric $g$. If we denote by $P^{k}$ the set of covariant 2-tensor fields obtained in this way, then it is clear that $P^{k} \subseteq Z^{k}$, and the question is whether there is an equality.

In this paper we prove that the orthogonal complement of $P^{k}$ in $Z^{k}$ (denoted by $Z^{k} / P^{k}$ ) consists of smooth tensor fields up to the boundary (see Theorem 44). As a corollary (see Corollary 3) we prove that this space is a finite-dimensional vector space. The above corollary had been proved independently by V.A. Sharafutdinov 13, but our result proves that the dimension of this space does not depend on $k$. This answers one of the problems posed by V.A. Sharafutdinov in [14].

The analogous question for lower order tensor fields was answered for metrics that are simple in the work of Mukhometov [8, 9] (see also Bernstein and Gerver [3]). Anikonov and Romanov [1, 2] have solved the case of 1-tensor fields. In the case of tensors of order 2, some partial answers have been given by Pestov and Sharafutdinov [10] in the case on negatively curved manifolds, which was extended to manifolds of small positive curvature by Sharafutdinov [11. In the two-dimensional case we mention a result by Sharafutdinov and Uhlmann [15] for simple manifolds with non-focal points.

The paper is organized as follows. In section 2 we prove that if $P^{\prime}$ is the transpose of $P$ with respect to some inner product defined in that section, then $P^{\prime} P$ is a pseudodifferential operator of order -1 in $\Omega$. Here we follow [16]. In this case we compute explicitly the principal symbol of $P^{\prime} P$. In section 3 , we define an elliptic pseudodifferential operator on $\Omega$, whose construction is analogous to the one used to produce an elliptic operator from an elliptic complex (see for example 6]). We use this operator in section 4 to prove our main theorems using the theory of pseudodifferential operators that satisfy Boutet de Monvel's transmission condition [5]. In this paper we follow the book by Chazarain and Piriou [4] on this subject. The main idea in this section is that although the operator constructed in section 3 cannot improve the regularity of distributions in $Z^{k} / P^{k}$ to be in $H^{k+1}(\Omega)$, a new operator, induced naturally on the boundary, is elliptic and does actually improve the regularity of elements in $Z^{k} / P^{k}$.

\section{The X-RAY TRAnsform of a 2-Tensor}

The results of this section were obtained in unpublished notes [16]. For the reader's convenience we provide its full exposition.

Let $\Omega$ be a bounded domain with smooth boundary $\partial \Omega$. The Riemannian metric $g=\left(g_{i j}\right)$ induces an inner product $\langle\cdot, \cdot\rangle_{T^{*} \Omega}$, on the cotangent bundle $T^{*} \Omega$, whose matrix is $g^{-1}=\left(g^{i j}\right)$. Consider the energy associated to the inner product 
in cotangent space, $H_{g}=\frac{1}{2} \sum_{i, j=1}^{n} g^{i j} \xi_{i} \xi_{j}$, and denote by $\Phi_{g}(t)$ the Hamiltonian flow associated to $H_{g}$. We define the sphere bundle of a manifold $\Omega$ as the set $S T^{*} \Omega=\left\{(z, \omega):(z, \omega) \in T^{*} \Omega, \sum_{i, j=1}^{n} g^{i j} \omega_{i} \omega_{j}=1\right\}$. We will denote by $(x(t), \xi(t))$ the bicharacteristic curves of $H$ on $S T^{*} \Omega$, whose projections to $\Omega$ are called the geodesics of the metric $g$. It will be convenient to parameterize geodesics according to the point and direction that they hit the boundary. It is natural, therefore, to consider the set of incoming geodesics, which is parametrized by the set

$$
S T^{*} \partial \Omega_{-}=\left\{(z, \omega):(z, \omega) \in S T^{*} \partial \Omega,\langle\omega, \nu(z)\rangle_{T^{*} \Omega} \leq 0\right\},
$$

where $\nu(z)$ is the outer unit normal to $\partial \Omega$. We introduce a measure on $S T^{*} \partial \Omega_{-}$, $d \mu(z, \omega)=\langle\omega, \nu(z)\rangle_{T^{*} \Omega} d \omega d S$, where $d S$ is the surface measure on $\partial \Omega$ and so is $d \omega$ in $\mathbb{S}_{z}^{n-1}=\left\{\omega:(z, \omega) \in S T^{*} \partial \Omega\right\}$. In particular, any bicharacteristic $(x(t), \xi(t))$, associated to $H_{g}$, will be written in the form $(x(t ; z, \omega), \xi(t ; z, \omega))$, and it satisfies $(x(0 ; z, \omega), \xi(0 ; z, \omega))=(z, \omega)$.

In this section of the paper we will work in the space of symmetric smooth covariant two tensors, that is to say, a tensor or the form $\omega=\sum_{i, j=1}^{n} \omega_{i j} d x^{i} \otimes d x^{j}$, where $\omega_{i j}=\omega_{j i}$ is a smooth function, and we endow this space with the inner product induced by the metric. Explicitly this means that if $f$ and $h$ are two symmetric two tensors, then its inner product is defined as

$$
(f, h)=\sum_{i, j, k, l=1}^{n} \int_{\Omega} f_{i j}(x) \bar{h}_{k l}(x) g^{i k}(x) g^{j l}(x) d V_{g}
$$

where $d V_{g}$ is the canonical volume form induced by $g$ on $\bar{\Omega}$.

Let us consider a smooth 2-tensor field $\omega=\sum_{i, j=1}^{n} f_{i j}(x) d x^{i} \otimes d x^{j} \in C_{0}^{\infty}(\Omega)$, which we extend to be a smooth compactly supported tensor field in $\mathbb{R}^{n}$, vanishing outside $\Omega$, and let us note that we can write its geodesic X-ray transform as the function on $S T^{*} \partial \Omega_{-}$, given by

$$
(P f)(z, \omega)=\sum_{i, j=1}^{n} \int_{\mathbb{R}} f^{i j}(x(t ; z, \omega)) \xi_{i}(t ; z, \omega) \xi_{j}(t ; z, \omega) d t
$$

where $f^{i j}$ is obtained from $f_{i j}$ by raising indices.

It is clear that $P f \in L^{2}\left(S T^{*} \partial \Omega_{-} ; d \mu\right)$, since $\partial \Omega$ is compact. It is a classical technique when dealing with the problem of inversion of the X-ray transform to compute $P^{\prime} P$, where $P^{\prime}$ is the transpose of $P$, with respect to the usual $L^{2}$ inner product in $L^{2}\left(S T^{*} \partial \Omega_{-}\right)$, and we will do that in what follows. In fact, if we write $S=S T^{*} \partial \Omega_{-}$, and $h$ is another smooth symmetric two tensor field supported in $\Omega$, then

$$
\begin{aligned}
& \left(P^{\prime} P f, h\right)_{L^{2}(\Omega)}=(P f, P h)_{L^{2}\left(S T^{*} \partial \Omega_{-}\right)}=\int_{S} \operatorname{Pf}(z, \omega) \overline{P h}(z, \omega) d \mu(z, \omega) \\
= & \sum_{i, j, k, l=1}^{n} \int_{S}\left(\int_{\mathbb{R}} f^{i j}(x(t)) \xi_{i}(t) \xi_{j}(t) d t\right)\left(\int_{\mathbb{R}} \overline{h^{k l}}(x(s)) \xi_{k}(s) \xi_{l}(s) d s\right) d \mu(z, \omega) .
\end{aligned}
$$

Now if we use that

$$
\int_{-\infty}^{\infty} f(t) d t=\int_{0}^{\infty} f(s+t) d t+\int_{0}^{\infty} f(s-t) d t
$$


for all $s \in \mathbb{R}$, it follows that the above equation (1) can be written as a sum of two integrals $I_{+}$and $I_{-}$, where

$I_{ \pm}=\sum_{i, j, k, l=1}^{n} \int_{S} \int_{\mathbb{R}} \int_{0}^{\infty} f^{i j}(x(s \pm t)) \xi_{i}(s \pm t) \xi_{j}(s \pm t) \overline{h^{k l}}(x(s)) \xi_{k}(s) \xi_{l}(s) d t d s d \mu(z, \omega)$.

Remember that bicharacteristics, although not explicitly in the equation above, are parametrized by $(z, \omega)$. Note that for any $z \in \partial \Omega$ and for any $\omega \in \mathbb{S}_{z}^{n-1}$, $(z, s)$ are global coordinates in $\Omega$, where $s>0$ is the arclength parameter for $x(s ; z, \omega)$. On the boundary $d x=\omega^{T} g^{-1} \nu(z) d S d s$. Introduce the new variable $\xi=t \omega$ on the boundary, then at the boundary $d \xi d x=t^{n-1} d t d \omega d x=$ $\omega^{T} g^{-1} \nu(z) t^{n-1} d t d \omega d z d s=t^{n-1} d t d s d \mu(z, \omega)$. Since the Hamiltonian flow preserves the measure, the same is true for any $(x, \xi) \in T^{*} \Omega$. We can write

$$
(x(s+t), \xi(s+t))=\Phi(1)(x(s), \xi(s)),
$$

where $t=|\xi(s)|_{g}$, that is to say, if $y=\exp _{x(s)} \xi$, then $y=x(s+t)$, where $t=|\xi|_{g}$. If we denote by $\delta(x, y)$ the geodesic distance between $x$ and $y$, then one can show, using simply connectedness of $\Omega$, that $\xi(s+t)=d_{y} \frac{1}{2} \delta^{2}(x, \cdot)$, therefore,

$$
\left(P^{\prime} P f, h\right)=2 \sum_{i, j, k, l=1}^{n} \int_{\Omega} \int_{\mathbb{R}^{n}} f^{i j}\left(\exp _{x} \xi\right) \frac{\partial \delta}{\partial y_{i}} \frac{\partial \delta}{\partial y_{j}} \overline{h^{k l}}(x) \frac{\xi_{k}}{\|\xi\|_{g}} \frac{\xi_{l}}{\|\xi\|_{g}} \frac{d \xi}{\|\xi\|_{g}^{n-1}} d x .
$$

If we perform the change of variables $y=\exp _{x} \xi$, then we get that $\xi /|\xi|_{g}=$ $-d_{x} \delta(x, y)$ and $\xi=-\frac{1}{2} d_{x} \delta^{2}(x, y)$. We conclude that the Jacobian of this change of variables is given by $|\operatorname{det}(d \xi / d y)|=\frac{1}{2^{n}}\left|\operatorname{det} \frac{\partial^{2}}{\partial x \partial y} \delta^{2}\right|$, and therefore we get that

$$
\left(P^{\prime} P f\right)_{i j}(x)=2 \sum_{k, l, p, q=1}^{n} \int_{\Omega} \frac{g^{k p}(y) f_{k l}(y) g^{l q}(y)}{\delta(x, y)^{n-1}} \frac{\partial \delta}{\partial y_{p}} \frac{\partial \delta}{\partial y_{q}} \frac{\partial \delta}{\partial x_{i}} \frac{\partial \delta}{\partial x_{j}} \frac{\left|\operatorname{det} \frac{\partial\left(\delta^{2} / 2\right)}{\partial x \partial y}\right|}{(\operatorname{det} g(x))^{1 / 2}} d y .
$$

Lemma 1. Assume that $g$ is a smooth simple metric in $\Omega$. Then if $x$ is close to $y$, we have

$$
\begin{aligned}
\delta^{2}(x, y) & =\sum_{i, j=1}^{n} G_{i j}^{(1)}(x, y)(x-y)_{i}(x-y)_{j}, \\
\frac{\partial^{2} \delta^{2}(x, y)}{\partial x_{j}} & =2 \sum_{i=1}^{n} G_{i j}^{(2)}(x, y)(x-y)_{i}, \\
\frac{\partial^{2} \delta^{2}(x, y)}{\partial x_{i} y_{j}} & =2 G_{i j}^{(3)}(x, y),
\end{aligned}
$$

where $G^{(1)}, G^{(2)}$ and $G^{(3)}$ are smooth functions in $\Omega \times \Omega$ and $G_{i j}^{(1)}(x, x)=G_{i j}^{(2)}(x, x)$ $=G_{i j}^{(3)}(x, x)=g_{i j}(x)$.

Proof. Choose the covector $\xi$, so that $y=\exp _{x} \xi$. Then $\delta^{2}(x, y)=|\xi|_{g^{-1}}^{2}$. From the Hamiltonian system, by the Taylor expansion argument we find that $y-x=$ $g^{-1}(x) \xi+O\left(|\xi|^{2}\right)$, that is to say, $\xi=g(x)(y-x)+O\left(|y-x|^{2}\right)$. This implies that $\delta^{2}$ vanishes at least at second order when $x=y$, and therefore by the Taylor expansion we get the first formula. The second and the third follow by differentiation.

Lemma 2. The operator $L=P^{\prime} P$ is a pseudodifferential operator of order -1 in $\Omega$. 
Proof. We have seen that $L$ is an integral operator with kernel $K(x, y)$, in fact, we have that $K_{i j}^{k l}(x, y)$ is given by

$$
2 \sum_{p, q=1}^{n} \frac{g^{p k}(y) g^{l q}(y)}{(\operatorname{det} g(x))^{1 / 2}} \frac{\left[\tilde{G}^{(2)}(x-y)\right]_{p}\left[\tilde{G}^{(2)}(x-y)\right]_{q}\left[G^{(2)}(x-y)\right]_{i}\left[G^{(2)}(x-y)\right]_{j}}{\left((x-y)^{T} G^{(1)}(x-y)\right)^{\frac{n-1}{2}+2}}\left|G^{(3)}\right|,
$$

where $\tilde{G}_{i j}^{(2)}(x, y)=G_{i j}^{(2)}(y, x),\left|G^{(3)}\right|=\left|\operatorname{det}\left(G_{i j}^{(3)}\right)\right|$, and we write $G$ for $G(x, y)$. Hence,

$$
K_{i j}^{k l}(x, y)=\widehat{M}_{i j}^{k l}(x, y, x-y),
$$

where $M_{i j}^{k l}(x, y, \xi)$ is given by

$$
\begin{aligned}
& M_{i j}^{k l}(x, y, \xi) \\
& \quad=\frac{2}{(2 \pi)^{n}} \int e^{i \xi \cdot z} \sum_{p, q=1}^{n} \frac{g^{p k}(y) g^{l q}(y)}{(\operatorname{det} g(x))^{1 / 2}} \frac{\left[\tilde{G}^{(2)} z\right]_{p}\left[\tilde{G}^{(2)} z\right]_{q}\left[G^{(2)} z\right]_{i}\left[G^{(2)} z\right]_{j}}{\left(z^{T} G^{(1)} z\right)^{\frac{n-1}{2}+2}}\left|G^{(3)}\right| d z .
\end{aligned}
$$

This last equation implies that $M_{i j}^{k l}$ is the inverse Fourier Transform of a homogeneous function of degree $1-n$, so that $M_{i j}^{k l}$ is a homogeneous function of degree -1 , which proves that $P^{\prime} P$ is a pseudodifferential operator of order -1 .

We can use the formulas obtained above to compute the principal symbol of $P^{\prime} P$, in fact, we just need to replace $G^{(1)}, G^{(2)}, \tilde{G}^{(2)}, G^{(3)}$ in the equation above by $g$. Making the change of variables $z=g^{-1 / 2} z^{\prime}$, and denoting $z^{\prime}$ again by $z$, we obtain

$$
\begin{gathered}
\sigma_{-1}\left(P^{\prime} P\right)_{i j}^{k l}(x, \xi)=M_{i j}^{k l}(x, x, \xi) \\
=\sum_{p, q=1}^{n} \frac{2}{(2 \pi)^{n}} \int e^{i g^{-1 / 2} \xi \cdot z} \frac{g^{p k}(x) g^{l q}(x)}{|z|^{n+3}}\left[g^{1 / 2} z\right]_{p}\left[g^{1 / 2} z\right]_{q}\left[g^{1 / 2} z\right]_{i}\left[g^{1 / 2} z\right]_{j} d z \\
=\frac{2}{(2 \pi)^{n}} \int e^{i g^{-1 / 2} \xi \cdot z} \frac{1}{|z|^{n+3}}\left[g^{-1 / 2} z\right]_{k}\left[g^{-1 / 2} z\right]_{l}\left[g^{1 / 2} z\right]_{i}\left[g^{1 / 2} z\right]_{j} d z .
\end{gathered}
$$

Therefore for any covariant 2-tensors $f_{i j}$, and a contravariant 2 tensor $h^{k l}$, we have that

$$
\sum_{i, j, k, l=1}^{n} \sigma_{-1}\left(P^{\prime} P\right)_{i j}^{k l}(x, \xi) f_{k l} \bar{h}^{i j}=\sum_{i, j, k, l=1}^{n} M_{i j k l}^{0}\left(g^{-1 / 2} \xi\right)\left(g^{-1 / 2} f g^{-1 / 2}\right)_{k l}\left(g^{1 / 2} \bar{h} g^{1 / 2}\right)_{i j},
$$

with

$$
M_{i j k l}^{0}(\xi)=\frac{2}{(2 \pi)^{n}} \int e^{i \xi \cdot z}|z|^{-n-3} z_{i} z_{j} z_{k} z_{l} d z .
$$

Summarizing, we have proved the following result.

Theorem 1. Let $\Omega$ be an open subset of $\mathbb{R}^{n}$, and $g$ a simple metric on $\bar{\Omega}$. Then $P^{\prime} P$ is a pseudodifferential operator of order -1 . If we denote by $\sigma_{-1}^{g}\left(P^{\prime} P\right)$ its 
principal symbol, then we have

$$
\begin{aligned}
& \sum_{i, j, k, l=1}^{n} \sigma_{-1}^{g}\left(P^{\prime} P\right)_{i j}^{k l}(x, \xi) f_{k l} \bar{h}^{i j} \\
& \quad=\sum_{i, j, k, l=1}^{n} \sigma_{-1}^{e}\left(P^{\prime} P\right)_{i j}^{k l}\left(g^{-1 / 2} \xi\right)\left(g^{-1 / 2} f g^{-1 / 2}\right)_{k l}\left(g^{1 / 2} \bar{h} g^{1 / 2}\right)_{i j} .
\end{aligned}
$$

The importance of the above calculation is that it relates the principal symbol of the operator $P^{\prime} P$ in a simple metric $g$ with the symbol of the operator $P^{\prime} P$ in Euclidean metric. Later in the paper we will use a formula analogous to equation (41) to define a new operator $L_{g}$, for any metric $g$, based on the construction of the operator $L_{e}$ defined for the Euclidean metric.

\section{A PARAMETRIX CONSTRUCTION}

In the last section we proved that $P^{\prime} P$ is a pseudodifferential operator of order -1 in $\Omega$. We will use the formula found in Theorem 1 as the basis of the construction of an elliptic pseudodifferential operator in $\Omega$. The construction is easily done in the case of the Euclidean X-ray transform and we will do so here; later we will use that construction to make a general construction for any metric.

Given a 1 -form $\omega$, we define its symmetric differential $d_{g}^{s} \omega$ as the symmetrization of the covariant derivative of $\omega$. In coordinates, this is defined as

$$
d_{g}^{s}\left(\sum_{i=1}^{n} a_{i} d x_{i}\right)=\sum_{i, j=1}^{n}\left[\frac{1}{2}\left(\frac{\partial a_{i}}{\partial x_{j}}+\frac{\partial a_{j}}{\partial x_{i}}\right)-\sum_{k=1}^{n} a_{k} \Gamma_{i j}^{k}\right] d x_{i} d x_{j} .
$$

The operator of symmetric differentiation has an adjoint with respect to the usual inner product in the space of symmetric 2 -tensor fields on $\Omega$ which we denote by $-\delta_{g}^{s}$. Here

$$
\delta_{g}^{s} f=\sum_{i=1}^{n} \sum_{j, k=1}^{n} g^{j k}\left(\frac{\partial f_{i j}}{\partial x_{k}}-\sum_{p=1}^{n} \Gamma_{j k}^{p} f_{p i}-\sum_{p=1}^{n} \Gamma_{i k}^{p} f_{p j}\right) d x^{i} .
$$

We will omit the index indicating the metric when the metric is Euclidean. In this case we have that the Laplacian, $\Delta=2 \delta^{s} d^{s}$, is given by

$$
\Delta\left(\sum_{j=1}^{n} a_{j} d x_{j}\right)=\sum_{i, j=1}^{n} \frac{\partial}{\partial x_{j}}\left(\frac{\partial a_{i}}{\partial x_{j}}+\frac{\partial a_{j}}{\partial x_{i}}\right) d x_{i} .
$$

Therefore its symbol is given by

$$
\sigma(\Delta)(\xi)=-\left(|\xi|^{2} I+\xi \xi^{T}\right),
$$

where $\xi=\left[\begin{array}{lll}\xi_{1} & \xi_{2} & \ldots \xi_{n}\end{array}\right]^{T}$ is a column matrix.

Note that $I-\Delta$ has symbol $\left(1+|\xi|^{2}\right) I+\xi \xi^{T}$, and that

$$
\left(\left(1+|\xi|^{2}\right) I+\xi \xi^{T}\right)^{-3 / 2}=\alpha\left(|\xi|^{2}\right) I+\beta\left(|\xi|^{2}\right) \xi \xi^{T}
$$

for some smooth functions $\alpha$ and $\beta$.

It is also easy to see that the symbol of the symmetric differential is given by

$$
B=\left[\begin{array}{lllll}
B_{1}^{T} & B_{2}^{T} & \ldots & B_{n}^{T}
\end{array}\right]^{T},
$$


where $B_{k}$ is an $n \times n$ matrix

$$
B_{k}=\frac{i}{2}\left(\xi_{k} I+\xi e_{k}^{T}\right),
$$

and finally for the divergence operator we have its symbol given by

$$
A=\left[\begin{array}{llll}
A_{1} & A_{2} & \ldots & A_{n}
\end{array}\right],
$$

where $A_{k}$ is an $n \times n$ matrix given by

$$
A_{k}=i e_{k} \xi^{T} \text {. }
$$

Here we have ordered the components of $f=\sum_{i, j=1}^{n} f_{i j} d x_{i} d x_{j}$ in a vector whose components are $\left(f_{11}, f_{12}, \ldots, f_{1 n}, f_{21}, f_{22}, \ldots, f_{2 n}, \ldots, f_{n 1}, f_{n 2}, \ldots, f_{n n}\right)^{T}$.

The main theorem of this section first appeared in [16].

Theorem 2. Let $(\bar{\Omega}, g)$ be a compact and simple Riemannian manifold. Then there exists an operator $M_{g}$, such that,

$$
C_{g}=P^{\prime} P+d_{g}^{s} M_{g} \delta_{g}^{s}
$$

is an operator of order -1 elliptic in $\Omega$.

Proof. First we prove the result in the case of the Euclidean metric. Here we can take $M_{e}=(I-\Delta)^{-3 / 2}$. Indeed, if $f$ is a tensor field in the kernel of $\sigma_{-1}\left(C_{e}\right)$, then

$$
\sigma_{-1}\left(P^{t} P\right) f+\sigma_{1}\left(d^{s}\right) \sigma_{-3}(I-\Delta)^{-3 / 2} \sigma_{1}\left(\delta^{s}\right) f=0 .
$$

Multiplying this equation by $\sigma_{1}\left(\delta^{s}\right)$ from the left, and using that $\sigma_{1}\left(\delta^{s}\right) \sigma_{-1}\left(P^{t} P\right) \equiv$ 0 , it follows that $\sigma_{2}\left(\delta d^{s}\right) \sigma_{-3}(I-\Delta)^{-3 / 2} \sigma_{1}\left(\delta^{s}\right) f=0$, so $\sigma_{1}\left(\delta^{s}\right) f=0$, due to the ellipticity of $\Delta(I-\Delta)^{-3 / 2}$.

From here and equation (9), it follows that $\sigma_{-1}\left(P^{\prime} P\right) f=0$. Therefore, all we need to do is to prove that if a tensor field satisfies the system of equations $\sigma_{1}\left(\delta^{s}\right) f=0$ and $\sigma_{-1}\left(P^{\prime} P\right) f=0$, then $f=0$.

Note first that the condition $\sigma_{1}\left(\delta^{s}\right) f=0$ is equivalent to

$$
\sum_{j=1}^{n} \xi_{j} f_{i j}=0, \text { for all } i=1, \ldots, n,
$$

which follows immediately from (6) and (7).

The principal symbol of $P^{\prime} P$ (given by (3)), can be explicitly computed, and it is given by (see equations 2.11.4 and 2.11.5 in [12])

$$
\sigma_{-1}\left(P^{\prime} P\right)_{i j}^{k l}(\xi)=C_{n} \partial_{i j k l}^{4}\|\xi\|^{3}
$$

for some non-zero constant $C_{n}$. The above expression can be explicitly computed by taking the fourth partial derivative of $\|\xi\|^{3}$. Due to (10) and the symmetry of $f$, when computing $\sigma_{-1}\left(P^{\prime} P\right)_{i j}^{k l} f_{k l}$, we can ignore any term in the principal symbol of $\sigma_{-1}\left(P^{\prime} P\right)$ that contains a factor $\xi_{k}$ or $\xi_{l}$. It follows that if $f$ is a tensor field such that $\sigma_{1}\left(\delta^{s}\right) f=0$ and $\sigma_{-1}\left(P^{\prime} P\right) f=0$, then

$$
\sum_{k, l=1}^{n}\left(\frac{\delta_{i j} \delta_{k l}}{\|\xi\|}+\frac{\delta_{i k} \delta_{j l}}{\|\xi\|}+\frac{\delta_{j k} \delta_{i l}}{\|\xi\|}-\frac{\xi_{i} \xi_{j} \delta_{k l}}{\|\xi\|^{3}}\right) f_{k l}=0, \text { for all } i, j=1, \ldots, n .
$$

If we choose $i=j$ in the above equation, and take the sum from $i=1$ to $n$, we obtain

$$
(n-1) \delta_{k l} f_{k l}=0
$$


from where it follows that

$$
\delta_{k l} f_{k l}=0 .
$$

Substituting this into equation (12), we get

$$
\sum_{k, l=1}^{n}\left(\frac{\delta_{i k} \delta_{j l}}{\|\xi\|}+\frac{\delta_{j k} \delta_{i l}}{\|\xi\|}\right) f_{k l}=0, \text { for all } i, j=1, \ldots, n
$$

which implies

$$
f_{i j}+f_{j i}=0, \text { for all } i, j=1, \ldots, n .
$$

Since $f$ is symmetric, it follows that $f=0$.

In order to end the proof of the theorem we need to construct $M_{g}$ for an arbitrary metric $g$, which will be done later (see Corollary 1). In order to start the construction of $M_{g}$, we need to compute the principal symbol of $L=d^{s}(I-\Delta)^{-3 / 2} \delta^{s}$.

Proposition 1. Let $L$ be the pseudodifferential operator in $\Omega$ of order -1 given by $L=d^{s}(I-\Delta)^{-3 / 2} \delta^{s}$; then the principal symbol of $L$ is

$$
\sigma_{-1}(L)_{i j}^{k l}(x, \xi)=-\frac{1}{2} \sum_{q=1}^{n}\left(\xi_{i} \delta_{j q}+\xi_{j} \delta_{i q}\right)\left(\alpha\left(|\xi|^{2}\right) \xi_{l} \delta_{q k}+\beta\left(|\xi|^{2}\right) \xi_{l} \xi_{q} \xi_{k}\right) .
$$

Proof. Let $f_{p q}$ be the components of a symmetric 2-tensor $f$ and let us note that $\sum_{k, l=1}^{n} \sigma\left(\delta_{e}\right)_{i}^{k l} f_{k l}=\sum_{l=1}^{n} \xi_{l} f_{i l}$. Hence, $\sum_{k, l=1}^{n} \sigma\left(\delta_{e}\right)^{k l} f_{k l}=\sum_{i, l=1}^{n} \xi_{l} f_{i l} e_{i}$, where $e_{i}$ is the vector whose only non-zero component is the $i$-th component, which is equal to one.

It follows that

$$
\sigma\left(\left(I+\delta d^{s}\right)^{-3 / 2}\right) \sigma\left(\delta^{s}\right)^{k l} f_{k l}=\alpha\left(|\xi|^{2}\right) \sum_{l, i=1}^{n} \xi_{l} f_{i l} e_{i}+\beta\left(|\xi|^{2}\right) \sum_{i, l=1}^{n} \xi_{i} \xi_{l} f_{i l} \xi .
$$

The proof is concluded by replacing the symbol of the symmetric differential in the above equation, and this yields equation (13). This concludes the proof.

As an important remark, note that the summand in (13) is a product of two terms. The first one corresponds to the symmetric differential, the other one to some pseudodifferential operator of order -2 .

Proposition 2. There exists a pseudodifferential operator $L_{g}$ on $\Omega$, such that $L_{e}=$ $L$ and

$$
\begin{aligned}
& \sum_{i, j, k, l=1}^{n} \sigma_{-1}\left(L_{g}\right)_{i j}^{k l}(x, \xi) f_{k l} \overline{h^{i j}} \\
& \quad=\sum_{i, j, k, l=1}^{n} \sigma_{-1}\left(L_{e}\right)_{i j}^{k l}\left(g^{-1 / 2} \xi\right)\left(g^{-1 / 2} f g^{-1 / 2}\right)_{k l}\left(g^{1 / 2} \bar{h} g^{1 / 2}\right)_{i j}
\end{aligned}
$$

for any covariant symmetric 2-tensor field $f$ and contravariant 2-tensor field $h$. The principal symbol $\sigma_{-1}\left(L_{g}\right)_{i j}^{k l}$, of $L_{g}$ is

(15) $\sum_{p=1}^{n}\left(\xi_{i}\left(G^{-1}\right)_{p j}+\xi_{j}\left(G^{-1}\right)_{p i}\right)\left(\alpha\left(|\xi|^{2}\right)\left(G^{2} \xi\right)_{l} G_{k p}+\beta\left(|\xi|^{2}\right)\left(G^{2} \xi\right)_{k}\left(G^{2} \xi\right)_{l}(G \xi)_{p}\right)$, where $G=g^{-1 / 2}$. 
Proof. Before we begin the proof, we note that the right-hand side in (13) is the sum of terms that have two factors. If we substitute (13) into (14), we realize that terms in the first factor in each summand are multiplied only by $h$, whereas the terms in the second factor are multiplied by $f$. Note that regardless of the changes we will make in equations (13) and (14), this will continue to be true.

In order to compute $\sigma_{-1}\left(L_{g}\right)_{i j}^{k l}(x, \xi)$, we need to simplify the expression

$$
\sum_{p, q, r, s=1}^{n} \sigma_{-1}\left(L_{e}\right)_{p q}^{r s}\left(g^{-1 / 2} \xi\right)\left(g^{-1 / 2} f g^{-1 / 2}\right)_{r s}\left(g^{1 / 2} h g^{1 / 2}\right)_{p q} .
$$

Let us analyze how each factor of (13) changes in each summand.

The first factor in (13) has the form (change $q$ by $\gamma$ in that equation)

$$
\xi_{p} \delta_{q \gamma}+\xi_{q} \delta_{p \gamma}
$$

so the term we need to consider is

$$
\begin{aligned}
I_{1} & =\sum_{p, q=1}^{n}\left(\left(g^{-1 / 2} \xi\right)_{p} \delta_{q \gamma}+\left(g^{-1 / 2} \xi\right)_{q} \delta_{p \gamma}\right)\left(g^{1 / 2} h g^{1 / 2}\right)_{p q} \\
& =\sum_{p, q, i, j=1}^{n}\left(\left(g^{-1 / 2} \xi\right)_{p} \delta_{q \gamma}+\left(g^{-1 / 2} \xi\right)_{q} \delta_{p \gamma}\right)\left(g^{1 / 2}\right)_{p i} h^{i j}\left(g^{1 / 2}\right)_{j q} .
\end{aligned}
$$

This expression simplifies to

$$
I_{1}=\sum_{i, j=1}^{n}\left(\xi_{i}\left(g^{1 / 2}\right)_{j \gamma}+\xi_{j}\left(g^{1 / 2}\right)_{i \gamma}\right) h^{i j},
$$

which gives the first factor in (15). In order to obtain the second factor we apply the same procedure. If we write $G=g^{-1 / 2}$, we obtain

$$
\begin{aligned}
I_{2} & =\sum_{r, s=1}^{n}\left(\alpha\left(|G \xi|^{2}\right)(G \xi)_{s} \delta_{\gamma r}+\beta\left(|G \xi|^{2}\right)(G \xi)_{s}(G \xi)_{\gamma}(G \xi)_{r}\right)(G f G)_{r s} \\
& =\sum_{r, s, k, l=1}^{n}\left(\alpha\left(|\xi|_{g^{-1}}^{2}\right)(G \xi)_{s} \delta_{\gamma r}+\beta\left(|\xi|_{g^{-1}}^{2}\right)(G \xi)_{s}(G \xi)_{\gamma}(G \xi)_{r}\right) G_{r k} f_{k l} G_{l s} \\
& =\sum_{k, l=1}^{n}\left(\alpha\left(|\xi|_{g^{-1}}^{2}\right)\left(g^{-1} \xi\right)_{l} G_{\gamma k}+\beta\left(|\xi|_{g^{-1}}^{2}\right)\left(g^{-1} \xi\right)_{l}(G \xi)_{\gamma}\left(g^{-1} \xi\right)_{k}\right) f_{k l} .
\end{aligned}
$$

Combining the expressions for $I_{1}$ and $I_{2}$, we obtain the result.

Note that $L_{g}$ is not uniquely determined by its principal symbol, we just need to find one pseudodifferential operator that satisfies equation (15). The principal symbol of $L_{g}$ can also be expressed as a sum of terms which factor in two terms. In particular the first factor in each summand corresponds to an operator, which we would like to compute explicitly. In order to do so, note that

$$
\begin{aligned}
\xi_{i}\left(G^{-1}\right)_{p j}+\xi_{j}\left(G^{-1}\right)_{p i} & =\sum_{q=1}^{n}\left(\xi_{i}\left(G^{-1}\right)_{p q} \delta_{q j}+\xi_{j}\left(G^{-1}\right)_{p q} \delta_{q i}\right) \\
& =\sum_{q=1}^{n}\left(\xi_{i} \delta_{q j}+\xi_{j} \delta_{q i}\right)\left(G^{-1}\right)_{p q} .
\end{aligned}
$$


If we move the factor $\left(G^{-1}\right)_{p q}$ to the second factor in the symbol of $L_{g}$ in (15), it is clear that we have proved, without loss of generality, that we can assume that the first factor in the principal symbol of $L_{g}$ corresponds to the symmetric differential operator $d$. Given that $d$ and $d_{g}^{s}$ have the same principal symbol, we can, without loss of generality, assume that $L_{g}$ factors from the left by $d_{g}^{s}$.

A similar analysis proves that $L_{g}$ can be factored from the right by $\delta_{g}^{s}$, although we will not use this fact later. We state it in the next corollary for future reference, and leave the proof to the reader.

Corollary 1. Let $L_{g}$ be an operator on $\Omega$, whose principal symbol is defined in Proposition 2. Then we can choose $L_{g}$ in such a way that it can be factored as

$$
L_{g}=d_{g}^{s} M_{g} \delta_{g}^{s}
$$

where $M_{g}$ is a pseudodifferential operator of order -3 in $\Omega$, which can be chosen so that $M_{e}=(I-\Delta)^{-3 / 2}$.

We can finally finish the proof of Theorem 2

End of the proof of Theorem 2. Note that formulas (4) and (14) imply that the principal symbol of $C_{g}$ satisfies

$$
\sum_{i, j, k, l=1}^{n} \sigma_{-1}\left(C_{g}\right)_{i j}^{k l} f_{k l} \bar{h}^{i j}=\sum_{i, j, k, l=1}^{n} \sigma_{-1}\left(C_{e}\right)_{i j}^{k l}\left(g^{-1 / 2} \xi\right)\left(g^{-1 / 2} f g^{-1 / 2}\right)_{k l}\left(g^{1 / 2} \bar{h} g^{1 / 2}\right)_{i j} .
$$

From this and the ellipticity of $C_{e}$, it is clear that $C_{g}$ elliptic.

\section{Regularity of the kernel of the Geodesic X-RAY transform}

It is clear, due to elliptic regularity and (8), that if $f$ is a distribution in $\Omega$ such that $P_{\gamma} f=0$ for any geodesic $\gamma$ and such that $\delta_{g}^{s} f=0$ in $\Omega$, then $f$ must be smooth in $\Omega$. However, its restriction to the boundary could be a non-smooth distribution on $\partial \Omega$. We will now prove that $f$ is actually smooth up to the boundary. In order to start, we will invert equation (8) to find a decomposition of the indentity into a potential part plus a term which behaves as a solenoidal part, modulo regularizing.

Proposition 3. There exists a pseudodifferential operators $T$ of order 1 and smoothing respectively $R$ in $\Omega$ such that

$$
I=T P^{\prime} P+d_{g}^{s} S \delta_{g}^{s}+R
$$

where $S$ is a parametrix of $\delta_{g}^{s} d_{g}^{s}$.

Proof. In order to simplify the notation in this proof, we will use the following convention. If $P$ and $Q$ are two pseudodifferential operators, we write $P \equiv Q$ if $P-Q$ is a smoothing operator.

The key idea is to invert equation (8). To do so, we know from Theorem 2 that

$$
C_{g}=P^{\prime} P+d_{g}^{s} M_{g} \delta_{g}^{s}
$$

is an elliptic operator of order -1 in $\Omega$. Applying symmetric differentiation by the right of both sides of the equation, we obtain

$$
C_{g} d_{g}^{s}=P^{\prime} P d_{g}^{s}+d_{g}^{s} M_{g} \delta_{g}^{s} d_{g}^{s}
$$

However, the operator $\delta_{g}^{s} d_{g}^{s}$ is an elliptic operator which has a parametric $S$; therefore

$$
C_{g} d_{g}^{s} S \equiv P^{\prime} P d S+d_{g}^{s} M_{g} .
$$


This implies that

$$
d_{g}^{s} M_{g} \equiv C_{g} d_{g}^{s} S-P^{\prime} P d S
$$

In particular replacing this equation into (8), we obtain that

$$
C_{g} \equiv P^{\prime} P+C_{g} d_{g}^{s} S \delta_{g}^{s}-P^{\prime} P d_{g}^{s} S \delta_{g}^{s} .
$$

Finally note that since $C_{g}$ is an elliptic operator, it has a parametrix $T$. Applying $T$ on the left we obtain

$$
I \equiv T P^{\prime} P+d_{g}^{s} S \delta_{g}^{s}-T P^{\prime} P d_{g}^{s} S \delta_{g}^{s} .
$$

We will prove that the last term in the above decomposition of the identity is smoothing, which will end the proof of the proposition.

In fact, let $S_{D}$ be the Dirichlet inverse for the Laplacian $\Delta^{s}=\delta_{g}^{s} d_{g}^{s}$ in $\Omega$. That is to say, $S_{D}$ is defined as the operator that satisfies

$$
\begin{array}{ll}
\Delta^{s} S_{D} f & =f \text { on } \Omega, \\
S_{D} f & =0 \text { on } \partial \Omega .
\end{array}
$$

This operator exists since the Laplace operator $\Delta^{s}$ satisfies the Lopatinskii condition. Recall that since $\Delta^{s}$ is an elliptic operator it has a parametrix $S$, such that $\Delta^{s} S f-f \in C^{\infty}(\Omega)$. In particular $\Delta^{s}\left(S f-S_{D} f\right) \in C^{\infty}(\Omega)$, so that by elliptic regularity $S f-S_{D} f \in C^{\infty}(\Omega)$.

Finally, this implies that

$$
\begin{aligned}
T P^{\prime} P d_{g}^{s} S \delta_{g}^{s} & =T P^{\prime} P d_{g}^{s}\left(S-S_{D}\right) \delta_{g}^{s}+T P^{\prime} P d_{g}^{s} S_{D} \delta_{g}^{s} \\
& =T P^{\prime} P d_{g}^{s}\left(S-S_{D}\right) \delta_{g}^{s}
\end{aligned}
$$

is a regularizing operator, since $P d_{g}^{s} S_{D}=0$. This concludes the proof.

Observe that we can extend all pseudodifferential operators in Proposition 3 to a neighborhood of $\Omega$ by extending the metric $g$. The above result is still valid in this extended domain, if we make the extended metric simple in this extended domain. In particular, the operators $d_{g}^{s}$ and $S$, defined in the above proposition, satisfy the transmission condition in $\Omega$. For a study of the transmission condition, we refer the reader to the book by Chazarain and Piriou [4. As a consequence of this extension, we have the following.

Corollary 2. Let $f \in H^{k}(\Omega), k \geq 1$, be such that $P f=0$ and $\delta_{g}^{s} f=0$. Then $f$ has restrictions of all orders to the boundary $\partial \Omega$ of $\Omega$.

Proof. Let $f^{0}$ be the extension of $f$ to a neighborhood of $\Omega$, which is identically zero outside $\Omega$. Using (17) we find that

$$
f^{0}=d_{g}^{s} S \delta_{g}^{s} f^{0}+R f^{0}
$$

where $S$ is a parametrix for the Laplacian, and $R$ is a smoothing operator. Note that of the two terms on the right-hand side, the second has restrictions of all orders to the boundary, because it is smooth in a neighborhood of $\partial \Omega$. The first term has restrictions of all orders to the boundary, because $\delta_{g}^{s} f^{0}$ vanishes in $\Omega$, and the operator $d_{g}^{s} S$ obviously satisfies the transmission condition there. Now the result follows directly from Theorem 2.2 in Chapter 5 of [4].

Let us introduce the following notation. In $\Omega$ we will use semigeodesic coordinates $\left(x^{\prime}, x_{n}\right)$ and $x_{n}>0$ in $\Omega$. In these coordinates, the boundary of $\Omega$ is defined by the equation $x_{n}=0$. The most important property of this coordinate system is that the metric satisfies $g_{i n}=\delta_{i n}$ for $i=1, \ldots, n$, where $\delta_{i j}$ is the Krönecker delta. 
If $f$ is a distribution in $\Omega$, we will denote by $f\left(x^{\prime}, 0^{+}\right)$its restriction to the boundary. If $f \in H^{1}(\Omega)$, then $f\left(x^{\prime}, 0^{+}\right)$belongs to $H^{1 / 2}(\partial \Omega)$. We will denote by $R^{+}$the operator that maps $f$ to $f\left(x^{\prime}, 0^{+}\right)$, which extends in an obvious way to tensor fields. In particular, given a symmetric tensor field $\omega=\sum_{i, j} \omega_{i j} d x^{i} d x^{j}$, we denote by $R_{n}^{+}$the operator $R_{n}^{+} \omega=\sum_{i=1}^{n} R^{+} \omega_{i n} d x^{i}$.

Note that if we use semigeodesic coordinates in $\Omega$, and if $\delta_{g}^{s} f=0$ in $\Omega$, then $\delta_{g}^{s} f^{0}=\sum_{i=1}^{n} f_{i n}\left(x^{\prime}, 0^{+}\right) \otimes \delta_{0}\left(x_{n}\right) d x^{i}=R_{n}^{+} f \otimes \delta_{0}\left(x_{n}\right)$ (see for example Theorem 3.1.9 in [7]), where on the right-hand side of the last equation $\delta_{0}\left(x_{n}\right)$ is the Dirac delta distribution in $\mathbb{R}$ at the origin.

Using equation (17) we conclude that if $f$ is a distribution such that $P f=0$ and such that $\delta_{g}^{s} f=0$, then

$$
f^{0}=d_{g}^{s} S\left(\sum_{i=1}^{n} f_{i n}\left(x^{\prime}, 0^{+}\right) \otimes \delta_{0}\left(x_{n}\right)\right) d x^{i}+R f^{0} .
$$

Note that the second term is smooth in a neighborhood of $\Omega$, and therefore has a smooth restriction to the boundary. It is a well-known fact (see Theorem 2.4 in Chapter 5 of [4) that the restriction to the boundary of the first term acts as a pseudodifferential operator of order 0 on the distribution $\sum_{i=1}^{n} f_{i n}\left(x^{\prime}, 0^{+}\right) d x^{i}$. We will, nevertheless, need to compute this operator explicitly.

Definition 1. Let $f$ be a smooth tensor field of order 1 on $\partial \Omega$. We will denote by $A$ the operator on $\partial \Omega$ defined by

$$
A f=R_{n}^{+} d_{g}^{s} \tilde{S}\left(f \otimes \delta_{0}\left(x_{n}\right)\right),
$$

where $\tilde{S}$ is the operator whose full symbol is equal to the principal symbol of any parametrix of $\delta_{g}^{s} d_{g}^{s}$, and $\delta_{0}\left(x_{n}\right)$ is the Dirac delta distribution on the boundary of $\Omega$.

The following proposition gives us the most important properties of $A$.

Proposition 4. The operator $A$ is a pseudodifferential operator on $\partial \Omega$ of order 0 , and its principal symbol is given by

$$
\sigma_{0}(A)\left(x^{\prime}, \xi^{\prime}\right)=\left[\begin{array}{cc}
\frac{I_{n-1 \times n-1}}{2} & -\frac{i \xi^{\prime}}{4\left\|\xi^{\prime}\right\|_{g^{\prime}}} \\
-\frac{i\left(g^{\prime} \xi^{\prime}\right)^{T}}{4\left\|\xi^{\prime}\right\|_{g^{\prime}}} & \frac{1}{2}
\end{array}\right] .
$$

Proof. It is well known that $\delta_{g}^{s} d_{g}^{s}$ has principal symbol given by

so

$$
\sigma_{2}\left(\delta_{g}^{s} d_{g}^{s}\right)=-\frac{1}{2}\left(\|\xi\|_{g}^{2} I+\xi(g \xi)^{T}\right),
$$

$$
\sigma_{-2}(S)=-\frac{2}{\|\xi\|_{g}^{2}}\left(I-\frac{\xi(g \xi)^{T}}{2\|\xi\|_{g}^{2}}\right) .
$$

Now (5) implies, that $d_{g}^{s} S$ has principal symbol

$$
C=\left[\begin{array}{llll}
C_{1}^{T} & C_{2}^{T} & \ldots & C_{n}^{T}
\end{array}\right]^{T},
$$

where $C_{k}$ is the $n \times n$ matrix

$$
C_{k}=-\frac{i}{\|\xi\|_{g}^{2}}\left(\xi_{k} I+\xi e_{k}^{T}-\frac{\xi_{k} \xi(g \xi)^{T}}{\|\xi\|_{g}^{2}}\right) .
$$


If we write $\xi=\left(\xi^{\prime}, \xi_{n}\right)$ and use semigeodesic coordinates, then $\|\xi\|_{g}^{2}=\xi^{, T} g^{\prime} \xi^{\prime}+\xi_{n}^{2}$ for some positive definite matrix $g^{\prime}$. We will denote $\left\|\xi^{\prime}\right\|_{g^{\prime}}=\left(\xi^{, T} g^{\prime} \xi\right)^{1 / 2}$.

Therefore the principal symbol of $A$ is given by

$$
\frac{1}{2 \pi} \lim _{x_{n} \rightarrow 0^{+}} \int_{-\infty}^{\infty} e^{i x_{n} \xi_{n}} \frac{-i}{\|\xi\|_{g}^{2}}\left(\xi_{n} I+\xi e_{n}^{T}-\frac{\xi_{n} \xi(g \xi)^{T}}{\|\xi\|_{g}^{2}}\right) d \xi_{n} .
$$

The above integral can be computed by the method of residues, and in particular we obtain

$$
\sigma_{0}(A)\left(x^{\prime}, \xi^{\prime}\right)=\left[\begin{array}{cc}
\frac{I_{n-1 \times n-1}}{2} & -\frac{i \xi^{\prime}}{4\left\|\xi^{\prime}\right\|_{g^{\prime}}} \\
-\frac{i\left(g^{\prime} \xi^{\prime}\right)^{T}}{4\left\|\xi^{\prime}\right\|_{g^{\prime}}} & \frac{1}{2}
\end{array}\right]
$$

This concludes the proof of the proposition.

Corollary 3. The operator $I-A$ is an elliptic pseudodifferential operator of order 0 on $\partial \Omega$.

Proof. The proof is immediate. Indeed, the operator $I-A$ has principal symbol

$$
\sigma_{0}(I-A)\left(x^{\prime}, \xi^{\prime}\right)=\left[\begin{array}{cc}
\frac{I_{n-1 \times n-1}}{2} & \frac{i \xi^{\prime}}{4\left\|\xi^{\prime}\right\|_{g^{\prime}}} \\
\frac{i\left(g^{\prime} \xi^{\prime}\right)^{T}}{4\left\|\xi^{\prime}\right\|_{g^{\prime}}} & \frac{1}{2}
\end{array}\right],
$$

which is an invertible matrix, because it only has trivial kernel, as it can be easily computed.

Our main result now follows.

Theorem 3. Let $f \in H^{k}(\Omega), k \geq 1$, be such that $P f=0$ and $\delta_{g}^{s} f=0$; then $f \in C^{\infty}(\bar{\Omega})$.

Proof. It follows from Proposition 3 that $f=R f$, where $R$ is a smoothing operator, and from where it follows that $f$ is smooth in the interior of $\Omega$. By Corollary $2, f$ has restrictions of all orders to $\partial \Omega$. Equality (17) implies that

$$
f^{0}=d_{g}^{s} S \delta_{g}^{s} f^{0}+R f^{0}
$$

If we decompose $S=\tilde{S}+T$, where $\tilde{S}$ is the operator whose full symbol is equal to the principal symbol of $S$ and $T$ has order -3 , then since $T$ is the difference of two operators that satisfy the transmission condition in $\Omega$, it also satisfies the transmission condition there. If we apply $R_{n}^{+}$to both sides of (20) we get

$$
R_{n}^{+} f=A R_{n}^{+} f+R_{n}^{+} d_{g}^{s} T\left(f \otimes \delta_{0}\left(x_{n}\right)\right)+R_{n}^{+} R f^{0} .
$$

Now Theorem 2.4 of Chapter 5 in [4] (see also Remark 2.6 there) implies that

$$
(I-A) R_{n}^{+} f=R_{n}^{+} d_{g}^{s} T\left(R_{n}^{+} f \otimes \delta_{0}\left(x_{n}\right)\right)+R_{n}^{+} R f^{0} \in H^{k+1 / 2}(\partial \Omega) .
$$

It follows from Corollary 3 that $R_{n}^{+} f \in H^{k+1 / 2}(\partial \Omega)$. Using (18) and Theorem 2.4 of Chapter 5 in [4], we conclude that $f \in H^{k+1}(\bar{\Omega})$, which completes the proof.

It is well known (see for example 12 that there exist continuous operators $S: H^{k}(\Omega) \rightarrow H^{k+1}(\Omega)$ and $R: H^{k}(\Omega) \rightarrow H^{k}(\Omega)$ such that for any tensor field $f$ we have

$$
f=d S f+R f
$$


Moreover, $\left.S f\right|_{\partial \Omega}=0$ and $\delta_{g}^{s} R f=0$. We have proved that if $f$ belongs to the kernel of the geodesic X-ray transform, then $R f$ is smooth up to the boundary of $\Omega$. We will prove in the next proposition that $Z^{k}$ embeds continuously in $H^{k+1}(\bar{\Omega})$.

Proposition 5. Let $f \in H^{k}(\bar{\Omega})$ such that $P f=0$ and $\delta^{s} f=0$. Then $f \in$ $H^{k+1}(\bar{\Omega})$, and there exists a constant $C_{k}>0$, such that

$$
\|f\|_{k+1 ; \Omega} \leq C_{k}\|f\|_{k ; \Omega} .
$$

Proof. It follows from equation (21) that

$$
\left\|(I-A) R_{n}^{+} f\right\|_{k+1 / 2 ; \partial \Omega} \leq C_{k}\|f\|_{k ; \Omega} .
$$

Now by Corollary 3 the operator $I-A$ is elliptic, so that using a standard elliptic estimate it follows that

$$
\left\|R_{n}^{+} f\right\|_{k+1 / 2 ; \partial \Omega} \leq C_{k}\|f\|_{k ; \Omega} .
$$

Finally, if we use (18) and Theorem 2.4 and Remark 2.6 in [4, we conclude that there exists a positive constant $C_{k}$ such that

$$
\|f\|_{k+1 ; \Omega} \leq C_{k}\|f\|_{k ; \Omega} \text {. }
$$

Theorem 4. Let $k \geq 1$, let $P^{k}=\left\{f: f=d_{g}^{s} v,\left.v\right|_{\partial \Omega}=0, v \in H^{k}(\Omega)\right\}$, and let $Z^{k}=\left\{v \in H^{k}(\Omega): P v=0\right\}$. Then $P^{k} \subseteq Z^{k}$, and $P^{k}$ has finite codimension in $Z^{k}$.

Proof. Estimate (22) shows that a bounded sequence in $H^{k}(\bar{\Omega})$ is also bounded in $H^{k+1}(\bar{\Omega})$. Using the Reillich Lemma, such a sequence has a convergent subsequence in $H^{k}(\bar{\Omega})$. This proves that the unit ball in $Z^{k}(\bar{\Omega})$ is compact, which is equivalent to the fact that $Z^{k}(\bar{\Omega})$ has finite dimension.

\section{ACKNOWLEDGMENTS}

The author would like to express his gratitude to his advisor Gunther Uhlmann for all of his support while studying at the University of Washington. His support has been invaluable. The author would also like to thank Professor V.A. Sharafutdinov for his careful reading of earlier versions of this manuscript and for several helpful comments and discussions.

\section{REFERENCES}

[1] Yu. E. Anikonov Some Methods for the Study of Multidimensional Inverse Problems Nauka, Sibirsk Otdel., Novosibirsk (1978).

[2] Yu. E. Anikonov, V.G. Romanov On uniqueness of determination of a form of first degree by its integrals along geodesics. J. Inverse Ill-Posed Probl. 5, no. 6, 487-490 (1997). MR.1623603 (99f:53072)

[3] I.N. Bernstein, M.L. Gerver Conditions of distinguishability of metrics by hodographs Methods and algorithms of interpretation of seismological information. Computerized Seismology. Vol. 13. Nauka, Moscow 50-73 (1980).

[4] J. Chazarain, A. Piriou Introduction to the Theory of Linear Partial Differential Equations. Studies in Mathematics and its Applications. Volume 14. North-Holland Publishing Company. MR0678605 (83j:35001)

[5] L. Boutet de Monvel Boundary problems for pseudo-differential operators Acta Math. 126, 11-51 (1971). MR0407904 (53:11674)

[6] P. Gilkey The Index Theorem and the Heat Equation Princeton University (1974). MR0458504 (56:16704)

[7] L. Hörmander The Analysis of Linear Partial Differential Operators Grund. der Math. Wiss. 256, Springer-Verlag (1983) MR0717035 (85g:35002a) 
[8] R.G. Mukhometov On the Problem of Integral Geometry Math. Problems in Geophysics. Akad. Nauk. SSSR, Sibirsk. Otdel., Vychisl. Tsentr, Novosibirsk, 6, No. 2, 212-242 (1975).

[9] R.G. Mukhometov On a problem of reconstructing Riemannian metrics Sibirsk. Mat. Zh. 22, no. 3, 119-135, 237 (1981). MR.0621466 (82m:53071)

[10] L.N. Pestov, V.A. Sharafutdinov, Integral geometry of tensor fields on a manifold of negative curvature Siberian Math. J. 29, no. 3, 114-130 (1988). MR0953028 (89k:53066)

[11] V.A. Sharafutdinov, Integral geometry of a tensor field on a manifold whose curvature is bounded above Siberian Math. J. 33, no 3, 192-204 (1992). MR.1178471 (94d:53116)

[12] V.A. Sharafutdinov Integral Geometry of Tensor Fields Inverse and Ill-Posed Problems Series VSP (1994). MR1374572 (97h:53077)

[13] V.A. Sharafutdinov Finiteness theorem for the ray transform on Riemannian manifolds Inverse Problems 11, no. 5, 1039-1050 (1995). MR1353801 (97a:58186)

[14] V.A. Sharafutdinov Ray Transform on Riemannian Manifolds University of Washington (1999).

[15] V. Sharafutdinov and G. Uhlmann On deformation boundary rigidity and spectral rigidity of Riemannian surfaces with no focal points, J. Differential Geometry 56, 93-110 (2000). MR.1863022 (2002i:53056)

[16] P. Stefanov and G. Uhlmann Unpublished Notes, 1998.

Department of Mathematics, University of Washington, Seattle, Washington 98195

Current address: Department of Mathematical and Physical Sciences, Texas A\&M International University, Laredo, Texas 78041-1900 